\title{
The Silk Protein Sericin Promotes Viability of ARPE-19 and Induced Pluripotent Stem Cell-Derived Retinal Pigment Epithelial Cells In Vitro
}

\author{
Ayyad Zartasht Khan ${ }^{1,2}$, Tor Paaske Utheim ${ }^{2,3,4,5,6,7}$, Morten Carstens Moe ${ }^{1,5}$, Hans Christian D. Aass ${ }^{2}$, Dipak \\ Sapkota $^{3}$, Evan Michael Vallenari ${ }^{3}$, and Jon Roger Eidet ${ }^{5}$ \\ ${ }^{1}$ Institute of Clinical Medicine, Faculty of Medicine, University of Oslo, P.O. Box 1171 Blindern, 0318 Oslo, Norway; \\ ${ }^{2}$ Department of Medical Biochemistry, Oslo University Hospital, Kirkeveien 166, P.O. Box 4956, Nydalen, 0424 Oslo, Norway; \\ ${ }^{3}$ Department of Oral Biology, Faculty of Dentistry, University of Oslo, Sognsvannsveien 10, P.O. Box 1052, 0316 Oslo, Norway; \\ ${ }^{4}$ Department of Plastic and Reconstructive Surgery, Oslo University Hospital, Kirkeveien 166, P.O. Box 4956, Nydalen, 0424 Oslo, \\ Norway; \\ ${ }^{5}$ Department of Ophthalmology, Oslo University Hospital, Kirkeveien 166, P.O. Box 4956, Nydalen, 0424 Oslo, Norway; \\ ${ }^{6}$ Department of Clinical Medicine, Faculty of Medicine, University of Bergen, Bergen, Norway; \\ ${ }^{7}$ Department of Ophthalmology, Stavanger University Hospital, Stavanger, Norway.
}

Correspondence: Ayyad Z Khan (a.a.z.khan@studmed.uio.no), ORCID: https://orcid.org/00000002-2048-225X.

Conflict of interest statement: A patent application has been filed by the research group on the use of sericin in culture media (European Patent Application No. 16733583.5).

Funding Statement: This work was supported by grants from Norwegian Research Council (Forskerlinje-grant to AZK, 2014-2019); Futura Fond til vitenskapelig medisinsk forskning (grant to AZK, 2019-2020); and Fylkesmann H.B. Guldahl og hustru Lucy Guldahls legat til bekjempelse av kreft og andre alvorlige sykdommer (grant to AZK, 2019-2020).

Key words: Sericin, retinal pigment epithelium, viability, maturation, induced pluripotent stem-cell derived RPE, ARPE-19. 


\begin{abstract}
Purpose: Maintaining mature and viable retinal pigment epithelial cells (RPE) in vitro has proven challenging. Investigating compounds that can promote RPE-viability and maturation is motivated by RPE transplantation research, the quest to understand RPE physiology, and a desire to modulate RPE in pathological states. We have previously reported that the silk protein sericin promotes viability, maturation, and pigmentation of human fetal RPE. In the present study, our aim was to uncover whether these effects can be seen in adult retinal pigment epithelial cell line-19 (ARPE-19) and induced pluripotent stem cell-derived RPE (iPSC-RPE).

Methods: ARPE-19 and iPSC-RPE were cultured with or without $10 \mathrm{mg} / \mathrm{mL}$ sericin. After seven days, viability was assessed with calcein-acetoxymethyl ester (CAM) and ethidium homodimer-1 (EH-1) assays, flow cytometry, and morphometric analysis. Expression levels of RPE65, tyrosinase, and Pmel17 were quantified to compare maturation between the sericin-treated and control cultures. Light microscopy and staining of the tight junction protein zonula occludens protein $1(\mathrm{ZO}-1)$ were employed to study sericin's effects on RPE morphology. We also measured culture medium $\mathrm{pH}$, glucose, lactate, and extracellular ion content.

Results: Sericin-supplemented RPE cultures demonstrated significantly better viability compared to control cultures. Sericin appeared to improve ARPE-19 maturation and morphology in vitro. No effects were seen on RPE pigmentation with the concentration of sericin and duration of cell culture herein reported.
\end{abstract}

Conclusions: This is the first study to demonstrate that supplementing the culture media with sericin promotes viability of iPSC-RPE and ARPE-19. Sericin's viability-promoting effects may have important implications for retinal therapeutics and regenerative medicine research. 


\section{Introduction}

Investigating compounds that can promote retinal pigment epithelial cell (RPE) viability and maturation is motivated by RPE transplantation research, the quest to understand RPE physiology, and a desire to modulate RPE in pathological states. First, with regards to RPE transplantation, producing viable and mature RPE grafts in an efficient manner could decrease RPE production time, which today can take up to three months. ${ }^{1}$ Cutting production time could reduce the costs of cellbased therapy and the risk of infection and disease progression associated with prolonged culture. Second, a thorough understanding of the mechanisms behind RPE viability and maturation might provide valuable pathophysiological insight. Third, this knowledge can aid in our ability to pharmacologically control or restore the RPE layer in diseases where RPE dysfunction is thought to play a key role, such as age-related macular degeneration and selected retinal dystrophies. ${ }^{2}$ Hence, researching compounds that promote RPE viability and maturation is vital for the progression of retinal research and ocular regenerative medicine.

We recently discovered that the silk protein sericin promotes viability, melanogenesis, and maturation in human fetal RPE cells by activating the NF- $\mathrm{BB}$ pathway. ${ }^{3}$ As the reported study was carried out on primary fetal RPE cells only, the present study was undertaken to uncover whether these effects could be reproduced in other RPE types as well, such as the adult retinal pigment epithelial cell line-19 (ARPE-19) and induced pluripotent stem cell-derived retinal pigment epithelial cells (iPSC-RPE). ARPE-19 was included because it is by far the most common used RPE for experimental studies, and iPSC-RPE was included because stem cell-derived RPE are promising cell types in RPE cell-based therapeutics. ${ }^{4}$ By investigating these two RPE types in the present study, our aim was to uncover whether sericin's viability-, melanogenesis-, and maturation-promoting effects are limited to human fetal RPE only or extend to other RPE tissue as well. 


\section{Materials and Methods}

\subsection{Cell Culture Media and Reagents}

iPSC-RPE and ARPE-19 cells were obtained from Cellular Dynamics International Inc. (Madison, WI, USA) and the American Type Culture Collection (ATCC) (Manassas, VA), respectively. Minimum Essential Medium $\alpha($ MEM $\alpha)$, Gibco ${ }^{\mathrm{TM}}$ KnockOut $^{\mathrm{TM}}$ Serum Replacement $\left(\right.$ KnockOut $^{\mathrm{TM}}$ SR), N-2 Supplement, DMEM/F12, phosphate-buffered saline (FBS), Penicillin-Streptomycin, and Gentamicin were purchased from Thermo Fisher Scientific (Waltham, MA, USA). Hydrocortisone, taurine, sericin, and 3,3',5-Triiodo-L-thyronine were from Sigma Aldrich (St Louis, MO, USA). Vitronectin XFTM and CellAdhere ${ }^{\mathrm{TM}}$ Dilution Buffer were supplied by STEMCELL Technologies Inc. (Vancouver, Canada). Nunclon $\Delta$ surface 96 -well plates, pipettes and other routine plastics came from VWR International (West Chester, PA, USA). Rabbit polyclonal anti-tyrosinase, antipremelanosomal protein-17 (Pmel17), and mouse anti-zonula occludens-1 (ZO-1) antibodies were acquired from Santa Cruz Biotechnology (Dallas, TX, USA). Mouse monoclonal anti-RPE65, secondary fluorescein isothiocyanate (FITC) and cyanine 3 (Cy3) conjugated anti-mouse or antirabbit antibodies were from Abcam (Cambridge, UK). Live/dead viability assay were from Invitrogen (Carlsbad, CA, USA). Propidium iodide (PI) was purchased from Merck Norway (Oslo, Norway). Annexin V Fluoresceinisotiocyanat (FITC) and $10 \mathrm{x}$ annexin V buffer were purchased from Becton \& Dickinson (Oslo, Norway).

\subsection{Cell Culture}

Experiments were conducted on third passage iPSC-RPE cells that were shipped unpassaged from the supplier. ARPE-19 cells at passage 20 were acquired from ATCC and the cells were subjected to two to three additional passages at our lab in order to obtain sufficient number of cells for the experiments. iPSC-RPE cells were seeded $\left(30.000 \mathrm{cells} / \mathrm{cm}^{2}\right)$ on vitronectin-coated Nunclon $\Delta$ surface 96-well plates in a MEM $\alpha$-based medium consisting of $5 \%$ Knock-Out SR, $1 \% \mathrm{~N}-2$ 
supplement, $55 \mathrm{nM}$ hydrocortisone, $250 \mu \mathrm{g} / \mathrm{mL}$ taurine, $14 \mathrm{pg} / \mathrm{mL}$ triiodo-L-thyronine, and $25 \mu \mathrm{g} / \mathrm{mL}$ gentamycin. ARPE-19 cells were seeded $\left(5.000\right.$ cells $\left./ \mathrm{cm}^{2}\right)$ on regular Nunclon $\Delta$ surface 96 -well plates in a DMEM/F12-based medium supplemented with $100 \mathrm{U} / \mathrm{mL}$ penicillin, $0.1 \mathrm{mg} / \mathrm{mL}$ streptomycin, and FBS at a final concentration of 10\%. Both cell types were thawed from cryopreserved state and cultured under routine conditions of $95 \%$ air and $5 \% \mathrm{CO}_{2}$ at $37^{\circ} \mathrm{C}$ until $80 \%$ confluence and a high degree of intercellular contact (iPSC-RPE: day seven; ARPE-19: day four). Thereafter, cells were maintained for a total of seven days in culture media without or with sericin at a final concentration of $10 \mathrm{mg}$ sericin $/ \mathrm{mL}$ medium. Apart from addition of sericin, the sericinsupplemented media were identical to control media. All cultures were fed every two days by replacing the culture volume with an aliquot warmed to $37^{\circ} \mathrm{C}$.

\subsection{Live/Dead Viability Assay}

A combined calcein-acetoxymethyl ester (CAM) and ethidium homodimer-1 (EH-1) assay permitted quantification of cell viability and death. CAM is a non-fluorescent compound that is hydrolyzed to calcein (strongly fluorescent) by intracellular esterases in live cells. CAM can thus be used to detect live cells. EH-1 on the other hand, is a membrane impermeable compound that binds to DNA. Since dead and dying cells have damaged cell membranes (in contrast to healthy cells), EH-1 can be used as an indicator of cell death. ${ }^{5}$ At the end of the culture period, cell medium was aspirated and cells were incubated at $37^{\circ} \mathrm{C}$ for 30 minutes with PBS containing $1.0 \mu \mathrm{M} \mathrm{CAM}$ and $1.0 \mu \mathrm{M} \mathrm{EH}-1$. After washing the cultures three times with PBS, photomicrographs were captured at 200x magnification and subsequently analyzed using custom-made macros with ImageJ (National Institutes of Health, Bethesda, MD), as described previously. ${ }^{6}$

\subsection{Flow Cytometric Quantification of Viability}

Culture supernatant was collected and kept on ice. Cells were detached with trypsin with 2 minutes incubation at $37^{\circ} \mathrm{C}$, transferred to $1.5 \mathrm{~mL}$ Eppendorf tubes, washed twice with $0.5 \mathrm{~mL}$ PBS and 
centrifuged at $80 \mathrm{xg}$ for 4 minutes. Cell culture supernatant was then transferred back to corresponding cell samples. The cells were stained with $0.9 \mu \mathrm{g} / \mathrm{mL}$ annexin V FITC with $2.5 \mathrm{mM}$ $\mathrm{Ca}^{2+}(10 \mathrm{x}$ annexin $\mathrm{V}$ buffer). Five minutes prior to analysis, samples were stained with $8.3 \mu \mathrm{g} / \mathrm{ml} \mathrm{PI}$. Flow cytometric analysis of cell viability was performed with a BD Accuri C6 benchtop flow cytometer (Becton and \& Dickinson, Norway). The binding of annexin V FITC to exterior phosphatidyl serine (PS) was identified in the FL1 detector (filter 530/30). The binding of PI to nuclear DNA (the dye only enters dead cells that have permeable cell membrane) was detected in the FL3 detector (filter 616/23). Cell debris were excluded in a dot-plot of the area pulse of both forward and side scattered light (FSC-A and SSC-A). Doublets were excluded using pulse width FCS versus SCS-A. Annexin V negative and PI negative cells were defined as viable cells (lower left quadrant). Annexin V positive and PI negative cells were considered apoptotic (lower left quadrant). PI positive cells were considered dead (upper left and upper right quadrants).

\subsection{Quantitative Immunofluorescence}

Following the seven-day culture period, RPE cultures were stained with both DAPI and immunomarkers. Photomicrographs were captured at $200 \mathrm{x}$ original magnification at pre-determined locations in the culture wells using a Nikon Eclipse Ti fluorescence microscope with a DS-Qi1 blackand-white camera and a motorized stage. The exposure length and gain were maintained at a constant level for all samples and the fluorescence intensities of the FITC or Cy3 fluorochromes, which were conjugated to the secondary antibodies, were within the dynamic range of the camera. Immunofluorescence was quantified by custom-made macros for ImageJ in photomicrographs as described previously ${ }^{6}$, with some modifications. In brief, total fluorescence intensity (raw integrated density) was measured after subtracting for background fluorescence. To normalize for any difference in cell number between the photomicrographs, total fluorescence intensity was divided by the number of cells (DAPI count) in the same area in which total fluorescence intensity was measured. The same 
macro was used for all photomicrographs. This permitted an objective, reliable, and computerized immunofluorescence quantification.

\subsection{Immunoblotting}

Whole-cell lysates harvested with RIPA buffer and supplemented with protease inhibitor cocktail were used for western blot experiments. Briefly, $40 \mu \mathrm{g}$ total protein was loaded onto a 4-20\% gradient gel (Criterion TGX; Bio-Rad) and run at $120 \mathrm{~V}$ for 1 hour. Protein was transferred to a PVDF membrane by wet transfer. The blots were probed with antibodies for RPE65 (1:100; Santa Cruz sc390787), tyrosinase (1:200; Santa Cruz sc-20035), and glyceraldehyde 3-phosphate dehydrogenase (GAPDH; 1:1000; Santa Cruz sc-47724). GAPDH was used as loading control. The blots were visualized with either Clarity ${ }^{\mathrm{TM}}$ Western ECL substrate (Bio-Rad) or SuperSignal ${ }^{\mathrm{TM}}$ West Femto Maximum Sensitivity Substrate (Thermo Scientific).

\subsection{Quantitative Morphometric Analysis}

As apoptotic cells demonstrate distinctive morphometric features that can be objectively quantified ${ }^{7}$, DAPI-stained photomicrographs of RPE cultures were subjected to computerized morphometric analysis as described before. ${ }^{8}$

\subsection{Metabolic Analysis}

Media removed from culture vessels were subjected to metabolic analysis using a Radiometer ABL 700 blood gas machine (Bronshoj, Denmark). The analyzer was automatically calibrated following the manufacturer's protocol prior to analysis (Radiometer ABL 700 User Manual).

\subsection{Light Microscopy}

Three representative photomicrographs from each culture group were captured using a Leica DM microscope and Canon EOS 5D mark II camera at 200x magnification. 


\subsection{Statistical Analysis}

Except for immunoblotting, all experiments were performed as triplicates and repeated independently thrice. Two-sampled, two-tailed t-test in Microsoft ${ }^{\circledR}$ Excel ${ }^{\circledR}$ for Mac (ver. 16.16.19) was used to compare two groups. Data are expressed as mean \pm standard deviation of the mean. $P$-values were considered significant if $<0.05$.

\section{Results}

\subsection{Effects of Sericin on RPE Viability}

To examine the effects of sericin-supplementation on the viability of cultured RPE cells, we performed simultaneous CAM and EH-1-staining to quantify viability using fluorescence microscopy coupled with automated image analysis. Relative to control (set to $100 \%$ ), the area covered by live cells was significantly increased in sericin-treated RPE cultures (iPSC-RPE: $309 \% \pm 34 \%$; $p<0.001$, ARPE-19: $114 \% \pm 10 \% ; p=0.01)$. Significantly fewer EH-1-stained cells were found in sericinsupplemented cultures in comparison to untreated control (set to 100\%) (iPSC-RPE: 22\% \pm 8\%; $p<0.001$, ARPE-19: 4\% $\pm 4 \%$; $p<0.001$ ), indicating lower cell death. These results suggest better viability in sericin-treated RPE cultures in comparison to control cultures not supplemented with sericin (Figure 1).

We then employed flow cytometry to quantify the percentage of viable (annexin $\mathrm{V}$ negative \& PI negative), apoptotic (annexin V positive \& PI negative), and dead (PI positive) cells. While the percentage of viable cells was increased (iPSC-RPE cultured with sericin: $97 \% \pm 1 \%$; iPSC-RPE cultured without sericin: $94 \% \pm 1 \%$; $p<0.001$, ARPE-19 cultured with sericin: $97 \% \pm 1 \%$; ARPE-19 cultured without sericin: $83 \% \pm 1 \% ; p<0.001$ ), the percentage of apoptotic cells (iPSC-RPE cultured with sericin: $1 \% \pm 0 \%$; iPSC-RPE cultured without sericin: $1 \% \pm 1 \% ; p=0.047$, ARPE-19 cultured with sericin: $0 \% \pm 0 \%$; ARPE-19 cultured without sericin: $6 \% \pm 1 \% ; p<0.001$ ) and the percentage of dead cells (iPSC-RPE cultured with sericin: $2 \% \pm 1 \%$; iPSC-RPE cultured without sericin: 5\% $\pm 1 \%$; 
$p<0.001$, ARPE-19 cultured with sericin: $3 \% \pm 1 \%$; ARPE-19 cultured without sericin: $11 \% \pm 1 \%$; $p<0.001)$ were significantly reduced in sericin-supplemented cultures in comparison to control cultures. Thus, flow cytometry results were in line with CAM/EH-1 observations, suggesting better viability in sericin-supplemented RPE cultures.

In addition to CAM/EH-1 assays and flow cytometry experiments, we also analyzed nuclear morphometry as it has been reported to correlate with apoptosis. ${ }^{7}$ Nuclear area and nuclear circumference is negatively correlated with apoptosis, while nuclear form factor (in ImageJ termed circularity) correlates positively with apoptosis. ${ }^{7}$ Morphometric analysis revealed that, in comparison to control (set to $100 \%)$, mean nuclear circumference was reduced $(79 \% \pm 14 \% ; p=0.009)$, mean nuclear form factor was increased $(108 \% \pm 6 \% ; p=0.039)$, while mean nuclear area was unchanged $(72 \% \pm 30 \% ; p=0.052)$ in iPSC-RPE cultured with sericin. In ARPE-19 cultured with sericin, mean nuclear circumference $(131 \% \pm 20 \% ; p<0.001)$ and mean nuclear area $(155 \% \pm 35 \% ; p<0.001)$ was higher, while mean nuclear form factor was lower $(88 \% \pm 9 \% ; p=0.002)$ compared to control cultures. Thus, sericin-treated iPSC-RPE, but not ARPE-19, demonstrated morphometric changes consistent with less apoptosis.

\subsection{Effects of Sericin on RPE Maturation}

To assess sericin's effect on RPE maturation, we performed immunofluorescence experiments to quantify expression levels of RPE-specific proteins (Figure 2). Total fluorescence intensity was divided by the number of cells (DAPI count) to normalize for any difference in cell number between cultures. Relative to control (set to 100\%), ARPE-19 cells cultured in sericin-supplemented medium displayed significantly higher levels of the RPE-specific maturation markers RPE65 (142\% $\pm 40 \%$; $p=0.008)$, tyrosinase $(141 \% \pm 19 \% ; p<0.001)$, and Pmel17 $(156 \% \pm 18 \% ; p<0.001)$. The same tendencies were noted, although not at a statistically significant level, in iPSC-RPE (RPE65: 129\% \pm $60 \% ; p=0.18$, tyrosinase: $128 \% \pm 56 \% ; p=0.16)$. The immunofluorescence results show that supplementing ARPE-19 culture medium with sericin is associated with upregulation of markers consistent with RPE maturation. 
The presence of the RPE-specific markers RPE65 and tyrosinase was confirmed with immunoblotting (Figure 2).

\subsection{Effects of Sericin on RPE Morphology}

Next, we studied sericin's effects on RPE morphology. As RPE in vivo show a hexagonal cobblestone epithelial morphology, we employed light and immunofluorescence microscopy to study morphology and tight junction phenotype. Light microscopy demonstrated no clear difference between the supplemented and non-supplemented iPSC-RPE (Figure 3). In ARPE-19, however, the sericin-treated cells appeared less fusiform at culture day seven in comparison to control (Figure 4). Immunofluorescence staining of the tight junction protein ZO-1 revealed increased ZO-1 expression in ARPE-19 cultured with sericin compared to control $(125 \% \pm 17 \% ; p=0.01)$, while no significant difference was found in iPSC-RPE $(111 \% \pm 34 \% ; p=0.46)$. These two lines of evidence show that sericin improves ARPE-19 morphology following a seven-day culture period.

\subsection{Effects of Sericin on Culture Medium Metabolic Parameters}

To assess sericin's effects on metabolic parameters, we quantified the $\mathrm{pH}$, the partial pressure of $\mathrm{CO}_{2}$, the partial pressure of $\mathrm{O}_{2}$, bicarbonate, base excess (BE), anion gap (AG), glucose, and lactate in culture media at day zero (Table 1) and day seven of the culture period (Table 2). We found that media collected from sericin-supplemented RPE cultures had a significantly lower $\mathrm{pH}$ compared to control. This was accompanied by lower bicarbonate levels, lower BE, higher AG, lower glucose, and higher lactate in media collected from the sericin-supplemented cultures compared to control. The difference in glucose levels in iPSC-RPE, and the difference in lactate levels in ARPE-19 media were not statistically significant because glucose levels were too low to be detected (the machine is unable to quantify glucose levels below $1.0 \mathrm{mmol} / \mathrm{L}$ ) and lactate levels were above 20.0 (the machine is unable to quantify lactate levels above $20.0 \mathrm{mmol} / \mathrm{L}$ ). 


\subsection{Effects of Sericin on Extracellular Ion Content}

Because ion transport to and from neuroretina is one of the key functions of $\mathrm{RPE}^{4}$, we examined extracellular ion content in culture media collected at the start (Table 3) and end (Table 4) of the experiments. Except for a minor difference in chloride content in ARPE-19 media, sericinsupplemented media and the control media were similar in terms of ion content at the start of the experiments. At culture day seven, however, sericin-treated ARPE-19 cultures exhibited significantly lower levels of extracellular potassium and calcium, while extracellular levels of sodium and chloride were increased in comparison to untreated cultures. The same tendencies were noted, although not at a statistically significant level, in iPSC-RPE cultures. Even though the reasons remain unclear, these results show that sericin-treated RPE cultures had increased levels of extracellular sodium and chloride, and reduced levels of extracellular potassium and calcium, in comparison to control.

\section{Discussion}

In the present study, we explored the effects of sericin on cultured PPSC-RPE and ARPE-19. Culture media supplemented with $10 \mathrm{mg} / \mathrm{mL}$ sericin increased RPE viability in both iPSC-RPE and ARPE19 and promoted maturation of ARPE-19 cells. These results are in agreement with a previous study by the group ${ }^{3}$, in which sericin enhanced melanogenesis of cultured primary RPE through activation of the NF-kB pathway. Improved viability, maturation, and morphology was also demonstrated following storage of cultured primary RPE with sericin-supplemented storage medium. ${ }^{6}$ In addition to activation of the NF-kB pathway, sericin-induced maturation of cultured RPE could also be related to sericin's beneficial effect on viability. An RPE cell sheet with high viability usually have tightly adjoined cells. As RPE maturation is dependent on autocrine and paracrine stimulation ${ }^{9},{ }^{10}$, a high degree of confluence would potentially lead to stronger stimulation, and thus accelerated maturation. In support of the role of paracrine stimulation in RPE maturation, Rak et al reported that RPE appeared to become pigmented, i.e. more mature, in a clustered fashion. ${ }^{11}$ In the future, it would be 
worthwhile to explore molecular mechanisms behind our reported observations, and to what extent these can be used therapeutically.

In the experiments reported herein, we used a sericin concentration of $10 \mathrm{mg} / \mathrm{mL}$. The sericin concentration was chosen based on results from pilot studies on dose response, which suggested 10 $\mathrm{mg} / \mathrm{mL}$ to be the optimal final concentration of sericin in the culture medium. The duration of the culture period was based on previously published ${ }^{3}$ and unpublished data on fetal primary RPE, which have indicated that significant differences in viability and maturation can be observed after seven days of culture.

In addition to improving viability in both iPSC-RPE and ARPE-19 cultures, we demonstrate that the sericin-supplemented ARPE-19 cultures appeared more mature and showed significantly higher levels of RPE-specific maturation markers. Albeit elevated in sericin-treated iPSC-RPE, the differences in expression levels of RPE maturation markers were not statistically significant. Moreover, improvement in morphology was also not as readily apparent as in the ARPE-19 cultures. It is not unthinkable that one reason for not finding significant differences in maturation in the iPSCRPE cultures is that this cell type needs a longer culture period to become mature compared to the ARPE-19 cells. In fact, the supplier from which we acquired these cells recommends that these cells be cultured for 21 to 28 days. We deviated from these recommendations because we wanted to investigate whether the addition of sericin could accelerate the maturation process. Furthermore, keeping the duration of culture period unchanged gives us an opportunity to compare the effects of sericin on various types of RPE, which can become problematic if the culture duration is changed from cell type to cell type. We do acknowledge, however, that the cell types we discuss herein are vastly different. Not only that, the culture media also differ in great degree. For instance, the ARPE19 medium contained FBS, while the iPSC-RPE were cultured in FBS-free medium. We thus acknowledge that comparing the effects of sericin on two vastly different cells types cultured in markedly different culture media, is problematic. The present study should therefore be considered 
as a report on the effects of sericin on iPSC-RPE and ARPE-19 individually, and not as a comparison on the effects of sericin on iPSC-RPE versus ARPE-19.

Because animal models expressing all characteristics of various retinal diseases are lacking, studying the pathogenesis of these diseases is challenging. ${ }^{12}$ Additionally, patients with the same diagnosis often show differences in pathophysiology, and certainly show varying degree of improvement to the same treatment. ${ }^{4}$ Induced pluripotent stem cells (iPSC) can be used to develop a simple two-dimensional model to study patient-specific pathophysiology in patient-derived RPE. ${ }^{13}$, ${ }^{14}$ In the recent years, significant progress has been made in developing more efficient and less laborintensive protocols for generating differentiated RPE from iPSCs. ${ }^{15-18}$ For example, Maruotti and colleagues recently described a simple and efficient protocol for generating differentiated RPE from pluripotent stem cells without the use of growth factors. ${ }^{17}$ With such breakthroughs in streamlining iPSC-RPE protocols, it is hoped that in the future, one can harvest cells from individual patients, differentiate them into iPSC-RPE in vitro, study the RPE cells in the laboratory in order to understand pathogenesis specific to that patient or a group of patients, and thereby get a better chance of optimizing an individualized therapy. ${ }^{2,14}$ However, a critical first step towards generating such disease models is to maximize cell survival without the excessive use of growth factors (e.g. transforming growth factor beta and platelet-derived growth factor) that are known to cause epithelialto-mesenchymal transition in RPE during culture. ${ }^{19-21}$ As the data presented herein demonstrate, sericin could be used in culture media to improve iPSC-RPE viability without the use of additional growth factors.

When first reported, the ARPE-19 cell line showed characteristics of mature RPE; it exhibited a typical cobblestone morphology, expressed the RPE maturation markers CRALBP and RPE65, demonstrated adequate barrier functions, and appeared polarized. ${ }^{22}$ After two decades of passaging, however, the cell line has lost many of these features. ${ }^{4}$ In the recent years, much effort has therefore been devoted to identify factors that can promote ARPE-19 to regain a mature RPE phenotype. First, the passage of cells appears to be important in obtaining mature tissue. Geisen et al. reported that 
early passage ARPE-19 (defined as passages 15 to 17) were better in maintaining barrier function as measured by transepithelial resistance and ZO-1-staining in comparison to late passage cells (passages 32 to 34). ${ }^{23}$ Similarly, Samuel and colleagues also showed that low-passage ARPE-19 cells (passage 9 to 12 ) could be differentiated to express genes and phenotype similar to native RPE. ${ }^{24}$ Passages 22 to 28 were used to obtain polarized, phagocytosing, and even pigmented ARPE-19 cells by Ahmado et al. ${ }^{25}$ Second, culture media additives have an important role in promoting maturation. Nicotinamide $^{26}$, nicotinamide riboside ${ }^{26}$, and pyruvate ${ }^{25}$ have all been suggested as additives. The current experiments further present sericin as a culture media additive. Third, the total time in culture is important. Some investigators culture the cells for 14 days $^{23}$, some use a two month culture period ${ }^{26}$, while others maintain cells in culture for three ${ }^{25}$ and four ${ }^{24}$ months to obtain mature cells. Long culture periods carry disadvantages such as increased risk of contamination and epithelial-to mesenchymal transformation. ${ }^{4}$ Using sericin as a culture media additive may accelerate maturation and improve viability, as reported herein.

Regulation of extracellular $\mathrm{pH}$ by RPE is essential for a healthy retina. ${ }^{27}$ Analysis of culture media showed that media collected from sericin-supplemented RPE cultures had a significantly lower $\mathrm{pH}$ compared to control. However, the $\mathrm{pH}$ was well within the physiological range. This difference in $\mathrm{pH}$ was accompanied by lower bicarbonate levels, lower BE, higher AG, lower glucose, and higher lactate in media collected from the sericin-supplemented cultures. Since live cells produce lactate as a by-product of their energy metabolism ${ }^{28}$, and we, in the current experiments found higher viability in sericin-supplemented RPE cultures, we interpret the differences in $\mathrm{pH}$, bicarbonate, glucose, and lactate a reflection of the number of viable cells. In addition, because RPE is responsible for transporting high amounts of lactate produced by photoreceptor outer segments to the choroidal vasculature $^{29,30}$, we consider it beneficial, and important, that the sericin-treated cultures showed higher viability despite being exposed to elevated levels of lactate. Taken together, these data are interpreted as further evidence for better viability in sericin-supplemented RPE. 
Closely related to $\mathrm{pH}$ are the dynamics of sodium, potassium, calcium, and chloride. Sericintreated cultures showed lower levels of extracellular potassium and calcium, while extracellular levels of sodium and chloride were increased in comparison to untreated cultures. This pattern fits well with an increased activity of $\mathrm{Na}^{+} / \mathrm{K}^{+}$-ATPase, the chief ion transporter in RPE. The $\mathrm{Na}^{+} / \mathrm{K}^{+}$-ATPase shifts potassium intracellularly and transports sodium out of the cell, generating a sodium gradient that is used by the $\mathrm{Na}^{+} / 2 \mathrm{Cl}^{-} / \mathrm{K}^{+}$-co-transporter to accumulate chloride intracellularly. ${ }^{4}$ Importantly, $\mathrm{Na}^{+} / \mathrm{K}^{+}$ATPase-activity was not assessed in the current experiments, and statements concerning this pump must be considered speculations only.

The present study is limited by the lack of phagocytosis and transepithelial resistance, which may be interesting to study in future investigations. Phagocytosis of photoreceptor outer segments by RPE in vivo is a critical task as illustrated by the Royal College of Surgeons rat, in which retinal degeneration is thought to be caused by impaired RPE phagocytosis. ${ }^{31-33}$ Transepithelial resistance is $^{3}$ a method to assess the barrier function of RPE, which is important because RPE in vivo constitutes the outer blood retinal barrier. ${ }^{4}$ As both phagocytosis and high transepithelial resistance are features of a mature $\mathrm{RPE}^{4}$, the immunofluorescence data reported herein might give the reader an idea of the state of maturation. For example, the ZO-1 staining can, to some degree, be indicative of the barrier function. ${ }^{4}$ Despite the limitations, the findings reported herein might aid in improving RPE culture protocols.

In conclusion, this study demonstrates that supplementing the culture media with sericin promotes viability, and to some degree maturation, of RPE in vitro. While we have previously shown that sericin promotes viability and pigmentation in human fetal RPE, this is, to the best of our knowledge, the first time sericin's effects on ARPE-19 and iPSC-RPE are reported. The observations reported herein may have important implications for retinal therapeutics and regenerative medicine.

\section{Tables}




\begin{tabular}{|c|c|c|c|c|c|c|}
\hline \multirow[b]{3}{*}{$p H$} & \multicolumn{2}{|c|}{$\begin{array}{l}\text { iPSC-RPE } \\
\text { Mean } \pm \text { SD }\end{array}$} & \multicolumn{3}{|c|}{$\begin{array}{l}\text { ARPE-19 } \\
\text { Mean } \pm \text { SD }\end{array}$} & \multirow[t]{2}{*}{$P$-value } \\
\hline & Sericin $(10 \mathrm{mg} / \mathrm{mL})$ & Control & & Sericin $(10 \mathrm{mg} / \mathrm{mL})$ & Control & \\
\hline & $7.63 \pm 0.04$ & $7.67 \pm 0.05$ & 0.416 & $7.61 \pm 0.09$ & $7.72 \pm 0.12$ & 0.293 \\
\hline$p C O 2(k P a)$ & $1.47 \pm 0.13$ & $1.31 \pm 0.22$ & 0.352 & $1.71 \pm 0.42$ & $1.34 \pm 0.48$ & 0.374 \\
\hline$p O 2(k P a)$ & $24.10 \pm 0.48$ & $24.44 \pm 0.25$ & 0.329 & $23.87 \pm 0.43$ & $24.27 \pm 0.27$ & 0.245 \\
\hline $\begin{array}{l}\text { Bicarbonate } \\
(\mathrm{mmol} / \mathrm{L})\end{array}$ & $11.33 \pm 0.12$ & $10.93 \pm 0.45$ & 0.211 & $12.5 \pm 0.46$ & $12.2 \pm 0.69$ & 0.566 \\
\hline $\begin{array}{l}\text { BE } \\
(\mathrm{mmol} / \mathrm{L})\end{array}$ & $-9.70 \pm 0.79$ & $-9.47 \pm 0.5$ & 0.689 & $-8.83 \pm 1.01$ & $-7.47 \pm 1.32$ & 0.228 \\
\hline $\begin{array}{l}A G \\
(\mathrm{mmol} / \mathrm{L})\end{array}$ & $16.17 \pm 0.23$ & $16.6 \pm 0.56$ & 0.281 & $18.53 \pm 0.5$ & $19.27 \pm 0.32$ & 0.101 \\
\hline $\begin{array}{l}\text { Glucose } \\
(\mathrm{mmol} / \mathrm{L})\end{array}$ & $1.90 \pm 0.07$ & $1.9 \pm 0.01$ & 0.878 & $10.95 \pm 0.05$ & $11.17 \pm 0.19$ & 0.120 \\
\hline $\begin{array}{l}\text { Lactate } \\
(\mathrm{mmol} / \mathrm{L})\end{array}$ & $4.64 \pm 0.03$ & $4.51 \pm 0.05$ & 0.011 & $10.03 \pm 0.04$ & $10 \pm 0.21$ & 0.817 \\
\hline
\end{tabular}

Table 1. At the start of the experiments, no significant difference in $\mathrm{pH}$, the partial pressure of $\mathrm{CO}_{2}$, the

partial pressure of $\mathrm{O}_{2}$, bicarbonate, base excess (BE), anion gap (AG), glucose, or lactate in RPE culture media with and without sericin were found, except in iPSC-RPE culture media, where lactate levels were slightly (but significantly) elevated in the sericin-supplemented medium.

\begin{tabular}{|c|c|c|c|c|c|c|}
\hline & \multicolumn{6}{|c|}{ DAY SEVEN } \\
\hline & Mear & & $P$-value & Mean & & $P$-value \\
\hline & $\begin{array}{l}\text { Sericin (10 } \\
\mathrm{mg} / \mathrm{mL})\end{array}$ & Control & & $\begin{array}{c}\text { Sericin (10 } \\
\mathrm{mg} / \mathrm{mL})\end{array}$ & Control & \\
\hline$p H$ & $7.5 \pm 0.05$ & $7.59 \pm 0.03$ & 0.006 & $7.04 \pm 0.06$ & $7.42 \pm 0.05$ & $<0.001$ \\
\hline$p C O 2(k P a)$ & $1.81 \pm 0.33$ & $1.83 \pm 0.3$ & 0.907 & $1.23 \pm 0.24$ & $1.09 \pm 0.17$ & 0.2585 \\
\hline$p O 2(k P a)$ & $23.83 \pm 0.26$ & $24.15 \pm 0.3$ & 0.077 & $24.32 \pm 0.19$ & $24.35 \pm 0.23$ & 0.7691 \\
\hline $\begin{array}{l}\text { Bicarbonate } \\
(\mathrm{mmol} / \mathrm{L})\end{array}$ & $10.18 \pm 0.69$ & $12.75 \pm 1.99$ & 0.014 & $2.42 \pm 0.16$ & $5.05 \pm 0.18$ & $<0.001$ \\
\hline $\begin{array}{l}B E \\
(\mathrm{mmol} / \mathrm{L})\end{array}$ & $-12.95 \pm 0.28$ & $-9.08 \pm 2.17$ & 0.002 & $-28.22 \pm 0.84$ & $-19.52 \pm 0.69$ & $<0.001$ \\
\hline $\begin{array}{l}A G \\
(\mathrm{mmol} / \mathrm{L})\end{array}$ & $25.12 \pm 0.72$ & $16.82 \pm 2.69$ & $<0.001$ & $33.33 \pm 0.49$ & $26.55 \pm 0.1$ & $<0.001$ \\
\hline $\begin{array}{l}\text { Glucose } \\
(\mathrm{mmol} / \mathrm{L})\end{array}$ & 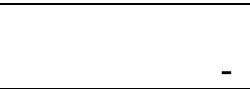 & $1.37 \pm 0.05$ & - & $4.39 \pm 0.06$ & $5.16 \pm 0.13$ & $<0.001$ \\
\hline $\begin{array}{l}\text { Lactate } \\
(\mathrm{mmol} / \mathrm{L})\end{array}$ & $5.84 \pm 0.23$ & $3.46 \pm 0.59$ & $<0.001$ & ‥ & $19.43 \pm 0.4$ & 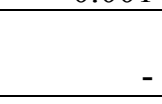 \\
\hline
\end{tabular}

Table 2. After seven days of culture, media collected from sericin-supplemented RPE had a significantly

lower $\mathrm{pH}$ compared to control. This was accompanied by lower bicarbonate levels, lower base excess (BE), higher anion gap (AG), lower glucose, and higher lactate in media collected from the sericin-supplemented cultures compared to control. The difference in glucose levels in iPSC-RPE, and the difference in lactate levels in ARPE-19 media were not statistically significant because glucose levels were too low to be detected (the machine is unable to quantify glucose levels below $1.0 \mathrm{mmol} / \mathrm{L}$ ) and lactate levels were above 20.0 (the machine is unable to quantify lactate levels above $20.0 \mathrm{mmol} / \mathrm{L}$ ). 


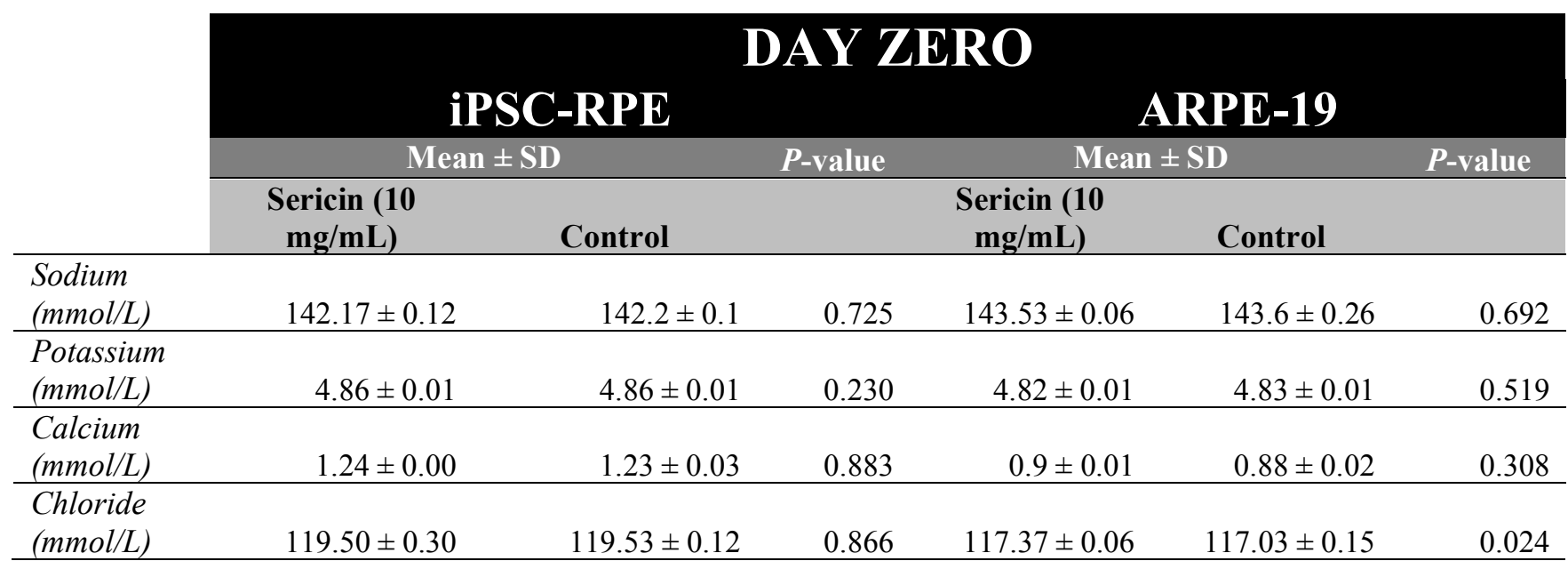

Table 3. Extracellular ion content was measured in all cultures at culture day zero. Except for a minor difference in chloride content in ARPE-19 media, sericin-supplemented media and the control media were similar in terms of ion content at the start of the experiments.

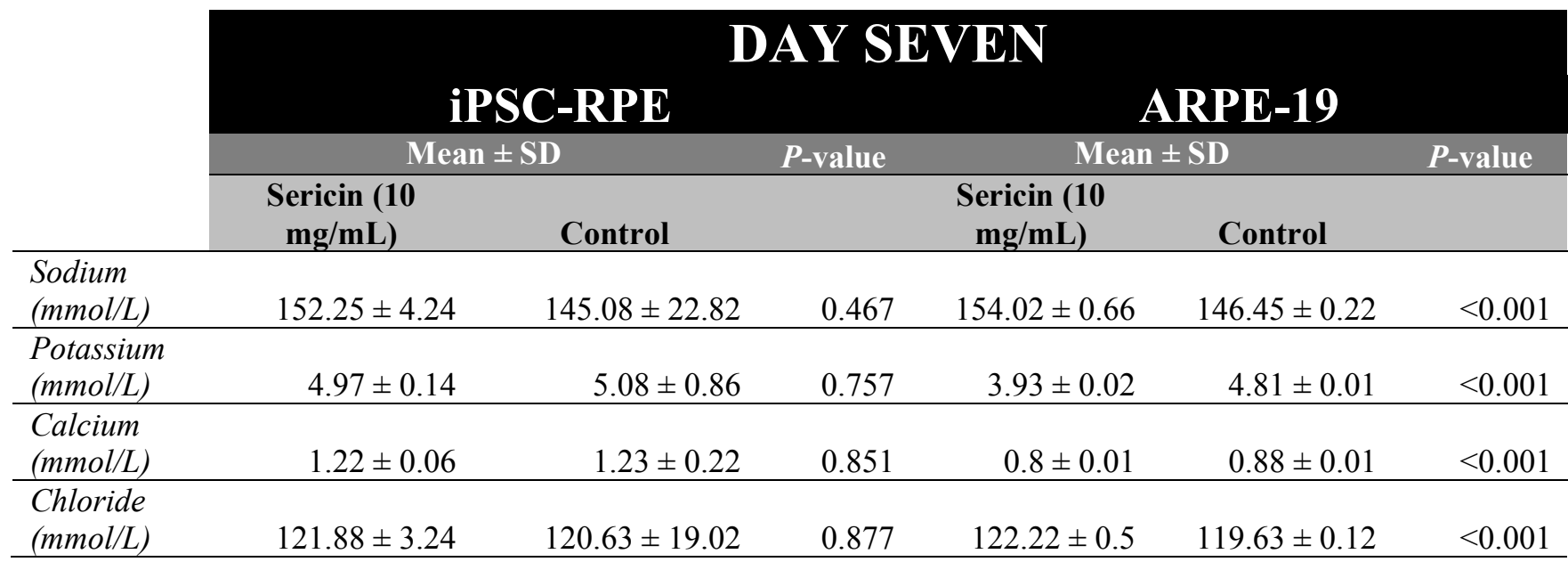

Table 4. Extracellular ion content was measured in all cultures at culture day seven. Sericin-treated ARPE-19 cultures exhibited significantly lower levels of extracellular potassium and calcium, while extracellular levels of sodium and chloride were decreased in comparison to untreated cultures. The same tendencies were noted, although not at a statistically significant level, in iPSC-RPE cultures. 


\section{Figure Titles}

Figure 1. Sericin-Supplemented RPE Cultures Show Significantly Higher Viability and Significantly Lower Cell Death Compared to Control Cultures.

Figure 2. Sericin-Supplemented RPE Cultures Show Higher Levels of RPE-Specific Maturation Markers Compared to Control Cultures.

Figure 3. Morphology of Induced Pluripotent Stem Cell-Derived Retinal Pigment Epithelial Cells (iPSC-RPE) Cultured in Sericin-Supplemented Medium.

Figure 4. Morphology of ARPE-19 Cells Cultured in Sericin-Supplemented Medium. 


\section{Figure Legends}

iPSC-RPE
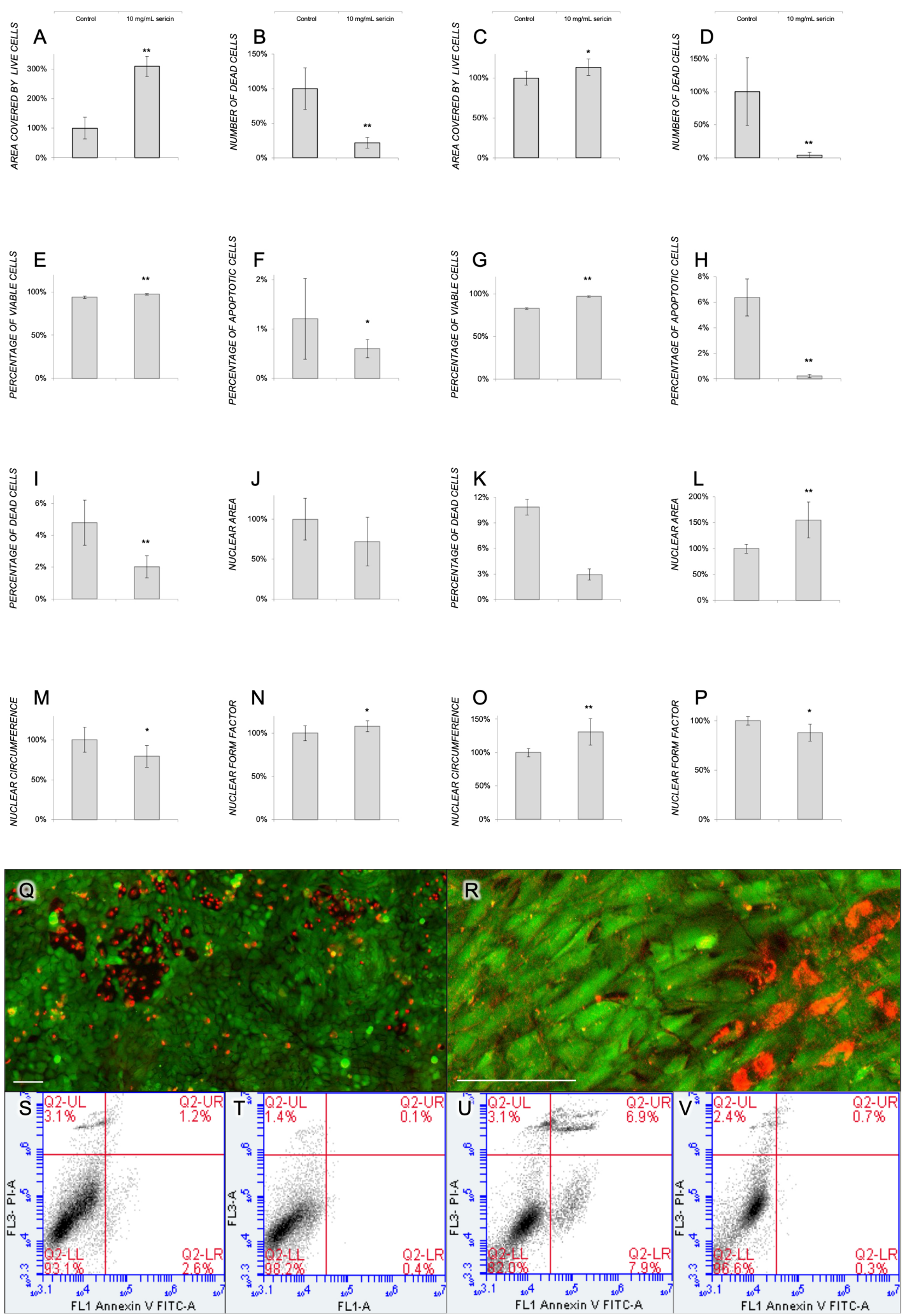

Figure 1. Sericin-Supplemented RPE Cultures Show Significantly Higher Viability and Significantly Lower Cell Death Compared to Control Cultures. Human induced pluripotent stem 
cell-derived retinal pigment epithelial cells (iPSC-RPE) and ARPE-19 cells were cultured for seven days in sericin-supplemented culture medium $(10 \mathrm{mg} / \mathrm{mL}$ sericin $)$. Cells cultured without addition of sericin to the medium served as control. Viability and cell death were quantified at the end of the culture period using immunofluorescence and flow cytometry. Mean nuclear area, nuclear circumference and nuclear form factor were objectively quantified by computerized analysis of photomicrographs of DAPI-stained nuclei. The bar charts show culture well area covered by calceinstained live iPSC-RPE (normalized to control) (A), number of EH-1-stained dead iPSC-RPE (normalized to control) (B), culture well area covered by calcein-stained live ARPE-19 (normalized to control) (C), number of EH-1-stained dead ARPE-19 (normalized to control) (D), percentage of viable iPSC-RPE (annexin V negative and PI negative) (E), percentage of apoptotic iPSC-RPE (annexin V positive and PI negative) (F), percentage of viable ARPE-19 (annexin V negative and PI negative) (G), percentage of apoptotic ARPE-19 (annexin V positive and PI negative) (H), percentage of dead iPSC-RPE (PI positive) (I), mean nuclear area of iPSC-RPE (normalized to control) (J), percentage of dead ARPE-19 (PI positive) (K), mean nuclear area of ARPE-19 (normalized to control) (L), mean nuclear circumference of iPSC-RPE (normalized to control) (M), mean nuclear form factor of iPSC-RPE (normalized to control) (N), mean nuclear circumference of ARPE-19 (normalized to control) (O), and mean nuclear form factor of ARPE-19 (normalized to control) (P). The bar charts show data from three independent repeats with each experiment done in triplicates and presented as mean values \pm standard deviation error bars. ${ }^{*} p<0.05 . * * p<0.001$. Photomicrographs from control cultures (not supplemented with sericin) show calcein-stained live (green) and EH-1stained dead (red) iPSC-RPE (Q) and ARPE-19 (R). Scale bars in Q and R are both $40 \mu \mathrm{m}$. Representative flow cytometry plots from iPSC-RPE cultured for seven days without (S) and with (T) sericin, and ARPE-19 cells cultured without (U) and with (V) sericin show percentage of annexin $\mathrm{V}$ negative and PI negative viable cells (lower left quadrant), annexin V positive and PI negative apoptotic cells (lower left quadrant), and PI positive dead cells (upper left and upper right quadrants). 


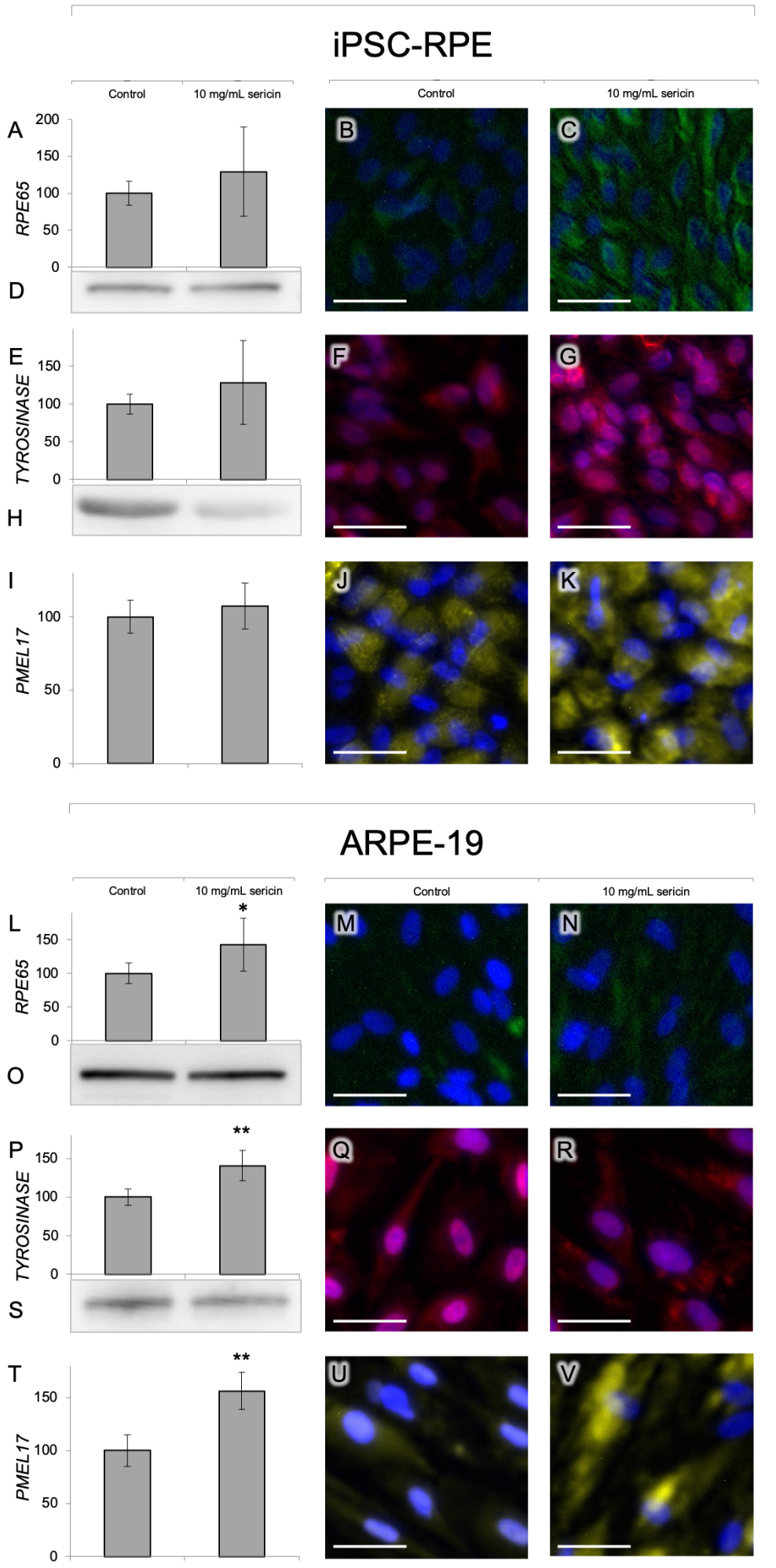

Figure 2. Sericin-Supplemented RPE Cultures Show Higher Levels of RPE-Specific Maturation

Markers Compared to Control Cultures. Human induced pluripotent stem cell-derived retinal pigment epithelial cells (iPSC-RPE) and ARPE-19 cells were cultured for seven days in sericinsupplemented culture medium. Cells cultured without addition of sericin to the medium served as 
control. The bar charts show total fluorescence intensity divided by cell count (DAPI count) of the RPE-specific markers RPE65 (A \& L), tyrosinase (E \& P), and Pmel17 (I \& T). Expression levels of these markers were significantly higher in sericin-supplemented ARPE-19 compared to control cultures (where the expression levels were set to $100 \%$ ). The same tendencies were noted, although not at a statistically significant level, in iPSC-RPE cultured with sericin. The bar charts show data from three independent repeats with each experiment done in triplicates and presented as mean values (normalized to control) \pm standard deviation error bars. ${ }^{*} p<0.05 .{ }^{*} * p<0.001$. Representative photomicrographs show anti-RPE65 (green), anti-tyrosinase (red), and anti-Pmel17 (yellow) in iPSCRPE (B, C, F, G, J, and K) and ARPE-19 (M, N, Q, R, U, and V) cultured with (C, G, K, N, R, and V) and without (B, F, J, M, Q, and U) sericin. Nuclei were stained with 4',6-diamidino-2-phenylindole (DAPI; blue). Scale bars are $30 \mu \mathrm{m}$. The presence of RPE65 and tyrosinase were confirmed with immunoblotting in iPSC-RPE (D \& H, respectively) and ARPE-19 (O \& S, respectively) cultured for seven days without and with $10 \mathrm{mg} / \mathrm{mL}$ sericin. 


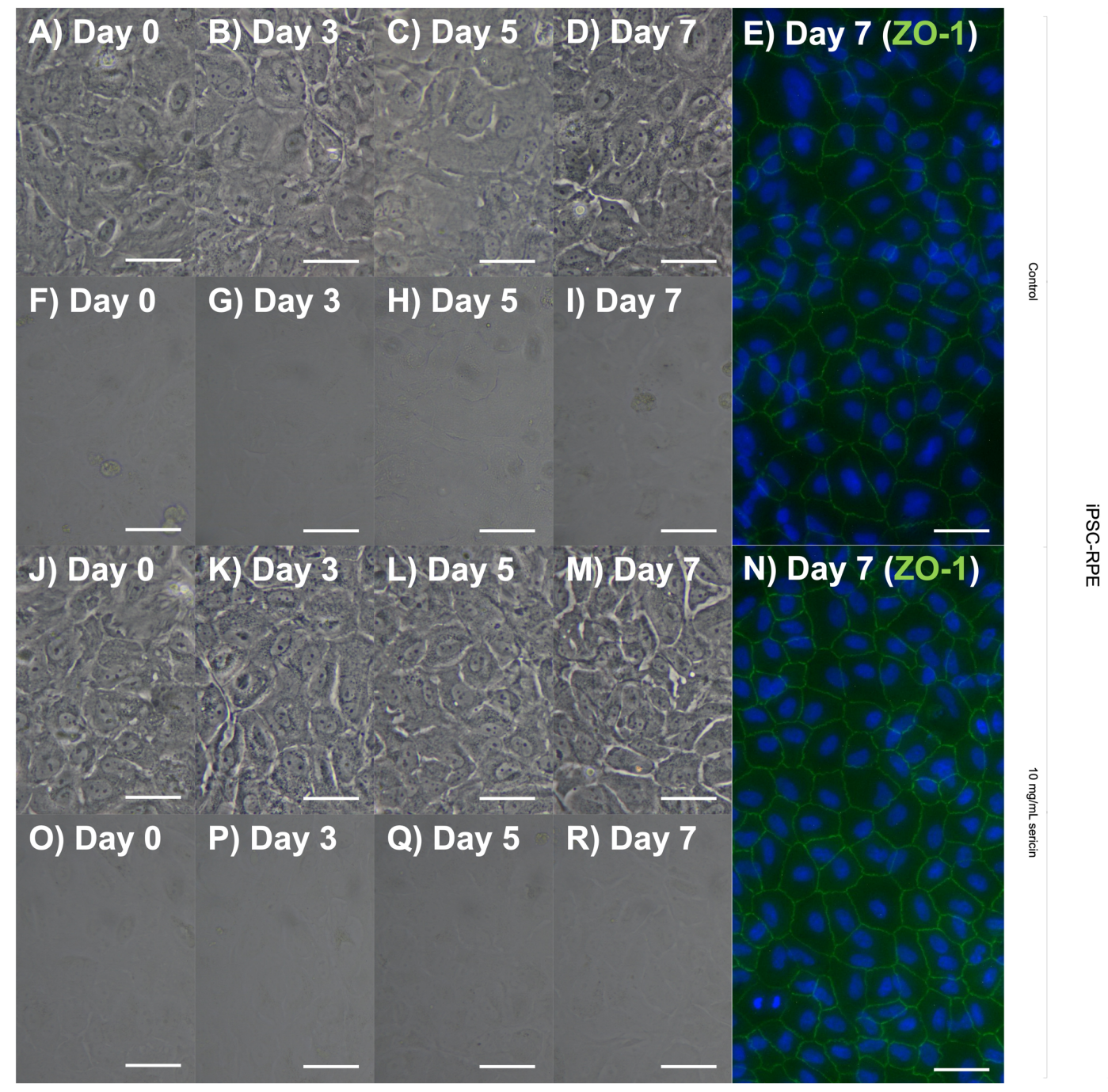

Figure 3. Morphology of Induced Pluripotent Stem Cell-Derived Retinal Pigment Epithelial Cells (iPSC-RPE) Cultured in Sericin-Supplemented Medium. iPSC-RPE were cultured for seven days in sericin-supplemented culture medium. Cells cultured without addition of sericin to the medium served as control. Light microscopy (F-I, O-R) and phase contrast microscopy (A-D, J-M) were employed to assess morphology at culture days zero, three, five, and seven. Immunofluorescence staining $(\mathrm{E}, \mathrm{N})$ of the tight junction protein ZO-1 (green) showed no significant difference between the sericin-supplemented cultures and control. Nuclei were stained with 4',6diamidino-2-phenylindole (DAPI; blue). Scale bars are $30 \mu \mathrm{m}$. 


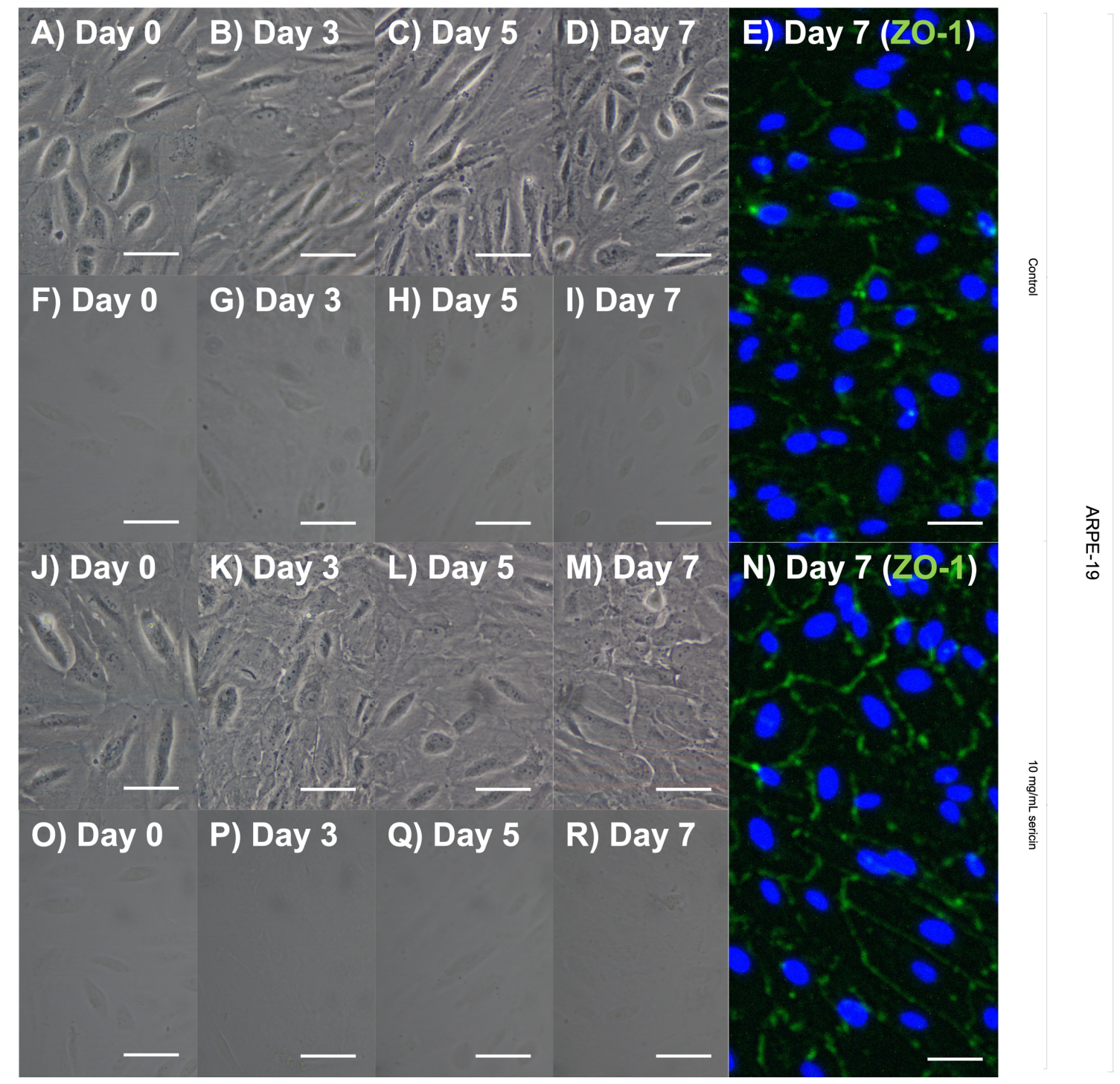

Figure 4. Morphology of ARPE-19 Cells Cultured in Sericin-Supplemented Medium. ARPE-19 cells were cultured for seven days in sericin-supplemented culture medium. Cells cultured without addition of sericin to the medium served as control. Light microscopy (F-I, O-R) and phase contrast microscopy (A-D, J-M) were employed to assess morphology at culture days zero, three, five, and seven. Less fusiform morphology was evident in sericin-supplemented ARPE-19 cells at day seven compared to control. Immunofluorescence staining (E, N) of the tight junction protein ZO-1 (green) showed significantly higher ZO-1 expression levels in the sericin-supplemented cultures compared to control. Nuclei were stained with 4',6-diamidino-2-phenylindole (DAPI; blue). Scale bars are 20 $\mu \mathrm{m}$. 


\section{Acknowledgements}

The authors thank Ms. Ingunn Rode Grorud, Senior Executive Officer at the Division of Laboratory Medicine, Faculty of Medicine, University of Oslo for her kind, flexible and timely logistical support.

\section{References}

1. Buchholz DE, Hikita ST, Rowland TJ, Friedrich AM, Hinman CR, Johnson LV, Clegg DO. Derivation of functional retinal pigmented epithelium from induced pluripotent stem cells. Stem cells 2009:27(10): 2427-2434.

2. Rathod R, Surendran H, Battu R, Desai J, Pal R. Induced pluripotent stem cells (ipsc)-derived retinal cells in disease modeling and regenerative medicine. J Chem Neuroanat 2019:95(8188.

3. Eidet JR, Reppe S, Pasovic L, Olstad OK, Lyberg T, Khan AZ, Fostad IG, Chen DF, Utheim TP. The silk-protein sericin induces rapid melanization of cultured primary human retinal pigment epithelial cells by activating the nf-kappab pathway. Sci Rep 2016:6(22671.

4. Klettner AK, Dithmar S. Retinal pigment epithelium in health and disease. Springer International Publishing.

5. Markovits J, Roques BP, Le Pecq JB. Ethidium dimer: A new reagent for the fluorimetric determination of nucleic acids. Anal Biochem 1979:94(2): 259-264.

6. Khan AZ, Utheim TP, Reppe S, Sandvik L, Lyberg T, Roald BBH, Ibrahim IB, Eidet JR. Cultured human retinal pigment epithelial (hrpe) sheets: A search for suitable storage conditions. Microscopy and Microanalysis 2018:24(2): 147-155.

7. Eidet JR, Pasovic L, Maria R, Jackson CJ, Utheim TP. Objective assessment of changes in nuclear morphology and cell distribution following induction of apoptosis. Diagn Pathol 2014:9(92.

8. Khan AZ, Utheim TP, Jackson CJ, Reppe S, Lyberg T, Eidet JR. Nucleus morphometry in cultured epithelial cells correlates with phenotype. Microsc Microanal 2016:22(3): 612-620.

9. Campochiaro PA, Hackett SF, Vinores SA, Freund J, Csaky C, LaRochelle W, Henderer J, Johnson M, Rodriguez IR, Friedman Z et al. Platelet-derived growth factor is an autocrine growth stimulator in retinal pigmented epithelial cells. J Cell Sci 1994:107 ( Pt 9)(24592469.

10. Liu ZZ, Zhu LQ, Eide FF. Critical role of trkb and brain-derived neurotrophic factor in the differentiation and survival of retinal pigment epithelium. The Journal of neuroscience : the official journal of the Society for Neuroscience 1997:17(22): 8749-8755.

11. Rak DJ, Hardy KM, Jaffe GJ, McKay BS. Ca++-switch induction of rpe differentiation. Exp Eye Res 2006:82(4): 648-656.

12. Song Min J, Bharti K. Looking into the future: Using induced pluripotent stem cells to build two and three dimensional ocular tissue for cell therapy and disease modeling. Brain Research 2016:1638(2-14.

13. Yang J, Li Y, Chan L, Tsai YT, Wu WH, Nguyen HV, Hsu CW, Li X, Brown LM, Egli D et al. Validation of genome-wide association study (gwas)-identified disease risk alleles with patient-specific stem cell lines. Hum Mol Genet 2014:23(13): 3445-3455.

14. Dalvi S, Galloway CA, Singh R. Pluripotent stem cells to model degenerative retinal diseases: The rpe perspective. Adv Exp Med Biol 2019:1186(1-31.

15. Zahabi A, Shahbazi E, Ahmadieh H, Hassani SN, Totonchi M, Taei A, Masoudi N, Ebrahimi 
M, Aghdami N, Seifinejad A et al. A new efficient protocol for directed differentiation of retinal pigmented epithelial cells from normal and retinal disease induced pluripotent stem cells. Stem Cells Dev 2012:21(12): 2262-2272.

16. Maruotti J, Wahlin K, Gorrell D, Bhutto I, Lutty G, Zack DJ. A simple and scalable process for the differentiation of retinal pigment epithelium from human pluripotent stem cells. Stem cells translational medicine 2013:2(5): 341-354.

17. Maruotti J, Sripathi SR, Bharti K, Fuller J, Wahlin KJ, Ranganathan V, Sluch VM, Berlinicke CA, Davis J, Kim C et al. Small-molecule-directed, efficient generation of retinal pigment epithelium from human pluripotent stem cells. Proceedings of the National Academy of Sciences 2015:112(35): 10950.

18. Smith EN, D'Antonio-Chronowska A, Greenwald WW, Borja V, Aguiar LR, Pogue R, Matsui $\mathrm{H}$, Benaglio $\mathrm{P}$, Borooah S, D'Antonio $\mathrm{M}$ et al. Human ipsc-derived retinal pigment epithelium: A model system for prioritizing and functionally characterizing causal variants at amd risk loci. Stem Cell Reports 2019:12(6): 1342-1353.

19. Massague J. Tgfbeta signalling in context. Nat Rev Mol Cell Biol 2012:13(10): 616-630.

20. Dvashi Z, Goldberg M, Adir O, Shapira M, Pollack A. Tgf- $\beta 1$ induced transdifferentiation of rpe cells is mediated by tak1. PloS one 2015:10(4): e0122229-e0122229.

21. Bastiaans J, van Meurs JC, van Holten-Neelen C, Nagtzaam NM, van Hagen PM, Chambers RC, Hooijkaas H, Dik WA. Thrombin induces epithelial-mesenchymal transition and collagen production by retinal pigment epithelial cells via autocrine pdgf-receptor signaling. Invest Ophthalmol Vis Sci 2013:54(13): 8306-8314.

22. Dunn KC, Aotaki-Keen AE, Putkey FR, Hjelmeland LM. Arpe-19, a human retinal pigment epithelial cell line with differentiated properties. Exp Eye Res 1996:62(2): 155-169.

23. Geisen P, McColm JR, King BM, Hartnett ME. Characterization of barrier properties and inducible vegf expression of several types of retinal pigment epithelium in medium-term culture. Curr Eye Res 2006:31(9): 739-748.

24. Samuel W, Jaworski C, Postnikova OA, Kutty RK, Duncan T, Tan LX, Poliakov E, Lakkaraju A, Redmond TM. Appropriately differentiated arpe-19 cells regain phenotype and gene expression profiles similar to those of native rpe cells. Mol Vis 2017:23(60-89.

25. Ahmado A, Carr AJ, Vugler AA, Semo M, Gias C, Lawrence JM, Chen LL, Chen FK, Turowski P, da Cruz L et al. Induction of differentiation by pyruvate and dmem in the human retinal pigment epithelium cell line arpe-19. Invest Ophthalmol Vis Sci 2011:52(10): 7148-7159.

26. Hazim RA, Volland S, Yen A, Burgess BL, Williams DS. Rapid differentiation of the human rpe cell line, arpe-19, induced by nicotinamide. Exp Eye Res 2019:179(18-24.

27. Strauss O. The retinal pigment epithelium in visual function. Physiol Rev 2005:85(3): 845-881.

28. Mather JP, Roberts PE. Introduction to cell and tissue culture: Theory and technique. Springer US.

29. Hsu SC, Molday RS. Glucose metabolism in photoreceptor outer segments. Its role in phototransduction and in nadph-requiring reactions. J Biol Chem 1994:269(27): 1795417959.

30. Bergersen L, Johannsson E, Veruki ML, Nagelhus EA, Halestrap A, Sejersted OM, Ottersen OP. Cellular and subcellular expression of monocarboxylate transporters in the pigment epithelium and retina of the rat. Neuroscience 1999:90(1): 319-331.

31. Dowling JE, Sidman RL. Inherited retinal dystrophy in the rat. J Cell Biol 1962:14(73-109.

32. Chaitin MH, Hall MO. Defective ingestion of rod outer segments by cultured dystrophic rat pigment epithelial cells. Invest Ophthalmol Vis Sci 1983:24(7): 812-820.

33. Mullen RJ, LaVail MM. Inherited retinal dystrophy: Primary defect in pigment epithelium determined with experimental rat chimeras. Science 1976:192(4241): 799-801. 